\title{
Top quark mass measurements with CMS
}

\author{
Nataliia Kovalchuk on behalf of the CMS Collaboration* \\ University of Hamburg, Luruper Chaussee 149, 22761 Hamburg, Germany \\ E-mail: nataliia.kovalchukedesy.de
}

\begin{abstract}
Measurements of the top quark mass are presented, obtained from CMS data collected in protonproton collisions at the LHC at centre-of-mass energies of 8 and $13 \mathrm{TeV}$. The mass of the top quark is measured using several methods and channels, including the reconstructed invariant mass distribution of the top quark, as well as measurements from shapes of top quark decay distributions. The results of the various channels are combined and compared to the world average. The dependence of the mass measurement on the kinematic phase space is investigated. The top mass and also $\alpha_{\mathrm{S}}$ are extracted from the top pair cross section measured at CMS.
\end{abstract}

XXV International Workshop on Deep-Inelastic Scattering and Related Subjects 3-7 April 2017

University of Birmingham, $U K$

\footnotetext{
* Speaker.
} 


\section{Introduction}

The measurement of the standard model parameters, such as the top quark mass, $\mathrm{m}_{\mathrm{t}}$, is an important part of the LHC physics program. Measuring precisely $\mathrm{m}_{\mathrm{t}}$ gives the opportunity to perform a consistency check of the standard model and can also be used to place constraints on new physics models. The most accurate value of the top quark mass was measured by the CMS Collaboration [1] using the Run 1 data and yields $m_{t}=172.35 \pm 0.51 \mathrm{GeV}$ [2]. Meanwhile, the results of $\mathrm{m}_{\mathrm{t}}$ coming from the ATLAS, CMS, CDF, and D0 experiments result in a world average mass of $\mathrm{m}_{\mathrm{t}}=173.34 \pm 0.27$ (stat) \pm 0.71 (syst) GeV [3]. The interpretation of the measured top quark mass is discussed in Refs. [4, 5, 6, 7, 8].

There are several approaches to measure $\mathrm{m}_{t}$ experimentally, namely direct and alternative methods. Where from one point of view, direct or "standard" ones give the most accurate value of the investigated quantity, from another point of view, alternative methods reduce the dependence on the detector calibration and test the stability of the results. In this work the latest CMS results for both mentioned methods at pp center-of-mass energies of $8 \mathrm{TeV}$ and $13 \mathrm{TeV}$ are presented.

\section{Direct measurements of $\mathbf{m}_{\mathrm{t}}$}

In the direct approach, the mass of the top quark is reconstructed by a kinematic fit of the decay products to a tit hypothesis. Since the top quark typically decays into a $\mathrm{W}$ boson and a $\mathrm{b}$ quark, where the $\mathrm{W}$ boson can decay either leptonicly or hadronicly, tree channels of the $t \bar{t}$ production are investigated: all-jets, $\overline{\mathrm{t}} \rightarrow \mathrm{WbWb} \rightarrow(\mathrm{q} \overline{\mathrm{q}} \mathrm{b})(\mathrm{q} \overline{\mathrm{q}} \mathrm{b})$, lepton+jets, $\overline{\mathrm{t}} \rightarrow \mathrm{WbWb} \rightarrow\left(1 v_{1} \mathrm{~b}\right)(\mathrm{q} \overline{\mathrm{q}} \mathrm{b})$ and dilepton, $\mathrm{t} \overline{\mathrm{t}} \rightarrow \mathrm{W}^{+} \mathrm{bW}-\mathrm{b} \rightarrow\left(1^{+} v_{1} \mathrm{~b}\right)\left(1^{-} v_{1} \mathrm{~b}\right)$. The all-jets decay mode has the highest rate and can be reconstructed completely, but it suffers from the much larger amount of background events coming from multijet processes. In contrast to this, the dilepton decay channel is characterized by a very pure signature, but has a very low rate and cannot

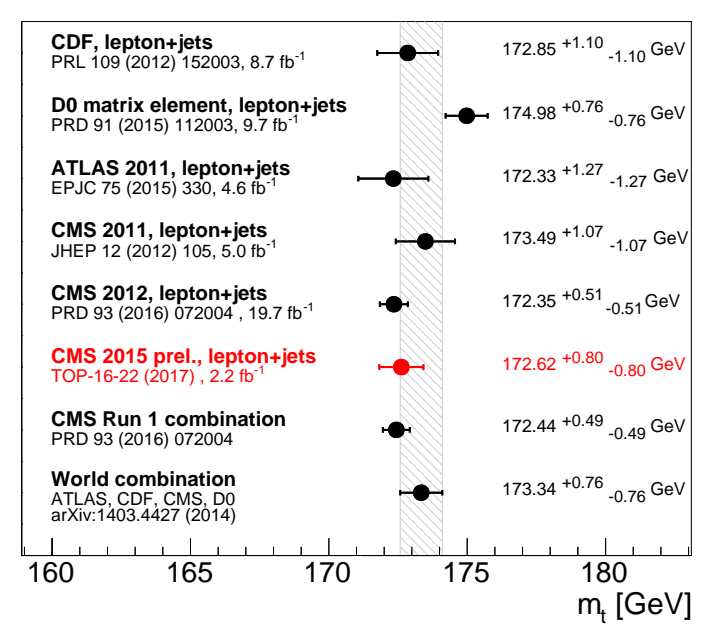

Figure 1: Comparison of Run 2 result (in red) [9] with previous measurements with lepton+jets final states [2, 10, 11, 12, 13] and the CMS [2] and world [3] averages. The $\mathrm{m}_{\mathrm{t}}$ measurements with lepton+jets final states. be fully reconstructed, due to presence of the neutrinos from both W bosons. Finally, the lepton+jets channel has fairly high rate and relatively low background contamination. In this case, the lepton helps during the selection of the events and it is possible to reconstruct the hadronically decaying top quark. More detail on the selection and reconstruction for these analyses can be found in Ref. [2]. A brief outline of the methodology is given below.

An analytical matrix-weighting technique (AMWT) is used in the dilepton channel to reconstruct the top quark. For each event, a scan of possible $\mathrm{m}_{\mathrm{t}}$ values from 100 to $600 \mathrm{GeV}$ is performed, 
taking into account up to 8 possible solutions of neutrino momenta. In addition, for each expected result a weight is assigned, that uses the probability of observing a charged lepton of energy $\mathrm{E}$ in a top frame [14]. Solutions with the highest average weight are named $\mathrm{m}_{\mathrm{t}}^{\text {AMWT }}$. The top quark mass is determined by comparing the $\mathrm{m}_{\mathrm{t}}^{\text {AMWT }}$ distribution to simulations for different $\mathrm{m}_{\mathrm{t}}$ hypotheses.

A kinematic fit and the ideogram method are used to measure $m_{t}$ in the all-jets and lepton+jets decay modes. A joint-likelihood fit to the selected events is employed to determine simultaneously the most probable top quark mass and jet energy scale factor (JSF). The fit takes into account all possible permutations per event. The kinematic fit to the $t \bar{t}$ hypothesis with the goodness-of-fit probability of $\mathrm{P}_{\text {gof }}>0.2$ is used to increase the fraction of correct permutations. The input variables to the fit are $\mathrm{m}_{\mathrm{t}}^{\mathrm{fit}}$ and the mass of the $\mathrm{W}$ boson in $\mathrm{q} \overline{\mathrm{q}}, \mathrm{m}_{\mathrm{W}}^{\text {reco }}$, that constrains the JSF. Thus, in Fig. 1 the results of $\mathrm{m}_{\mathrm{t}}$, measured in lepton+jets final state using Run 1 data, are summarized.
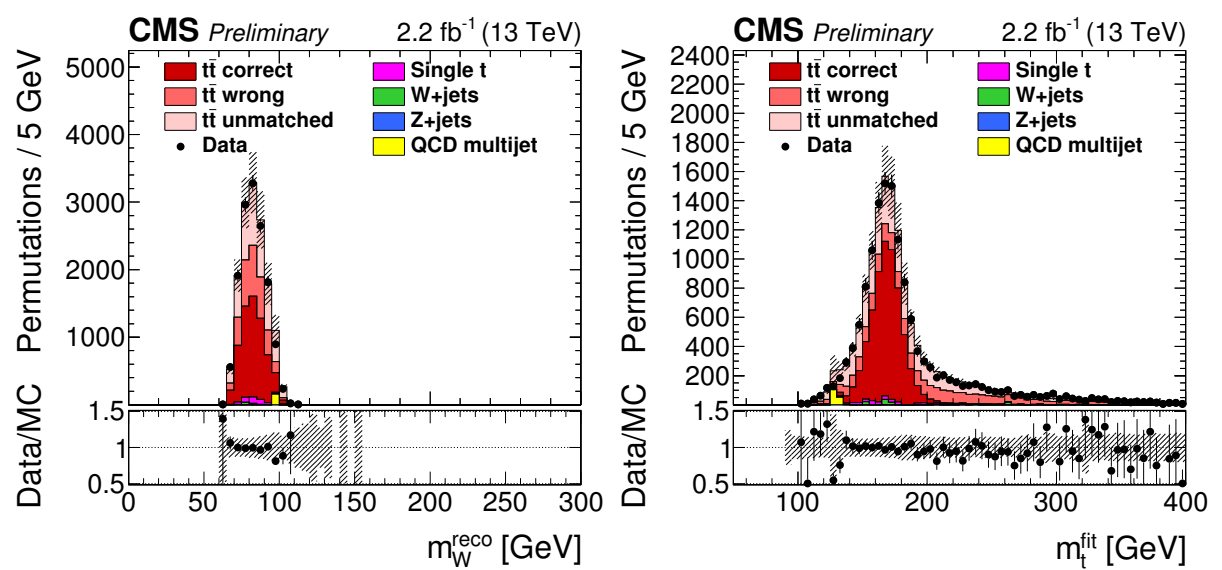

Figure 2: (left) The reconstructed $\mathrm{W}$ boson masses $\mathrm{m}_{\mathrm{W}}^{\text {reco }}$ and (right) the fitted top quark masses $\mathrm{m}_{\mathrm{t}}^{\mathrm{fit}}$ after the goodness-of-fit selection and the weighting by $\mathrm{P}_{\text {gof }}$ [9]. The vertical bars show the statistical uncertainty and the hatched bands show the statistical and systematic uncertainties added in quadrature. The lower portion of each panel shown the ratio of the yields between the collision data and the simulation.

The most recent analysis performed in lepton+jets channel at $13 \mathrm{TeV}$ [9], where the mass of the top quark is found to be $\mathrm{m}_{\mathrm{t}}^{\text {hyb }}=172.62 \pm 0.38$ (stat+JSF) \pm 0.70 (syst) GeV, is also shown in Fig. 1. The $\mathrm{m}_{\mathrm{t}}^{\text {fit }}$ and $\mathrm{m}_{\mathrm{W}}^{\text {reco }}$ distributions used in the analysis are presented in Fig. 2. The $\mathrm{m}_{\mathrm{t}}^{\text {hyb }}$ is calculated using the hybrid method, where a prior for the JSF from the jet energy correction uncertainty is used. The leading systematic uncertainty for this analysis comes from the jet energy correction, which is about $0.4 \mathrm{GeV}$. The results are consisten with ones at $8 \mathrm{TeV}$ [2] and confirm that there is no dependency of $\mathrm{m}_{\mathrm{t}}$ on the center-of-mass energy.

\subsection{Differential measurement of $m_{t}$ at $8 \mathrm{TeV}$}

Reference [2] provides results of the mass measurement in bins of different kinematic variables of the t $\bar{t}$ system, which is a good test of possible biases in different models for the soft and perturbative QCD, since they give the main contribution to the systematic uncertainties. Some examples of such a study are presented on the Fig. 3. No indications of a kinematical bias were observed. 

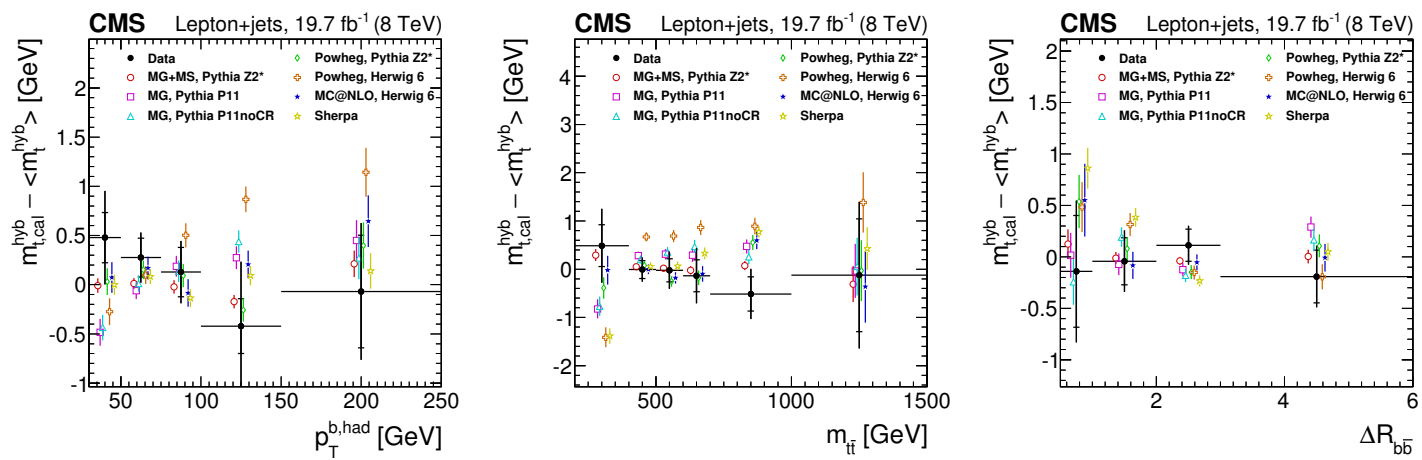

Figure 3: Measurements of $m_{t}$ as a function of the transverse momentum of the hadronically decaying top quark $\left(\mathrm{p}_{\mathrm{T}}^{\mathrm{t} \text {,had }}\right)$, the invariant mass of the $\mathrm{t} \overline{\mathrm{t}}$ system $\left(\mathrm{m}_{\mathrm{t}}\right)$ and the $\Delta R$ between the $\mathrm{b}$ jets $\left(\Delta \mathrm{R}_{\mathrm{b} \overline{\mathrm{b}}}\right)$ [2]. The filled circles represent the data, and the other symbols are for the simulations. For reasons of clarity the horizontal error bars are shown only for the data points and each of the simulations is shown as a single offset point with a vertical error bar representing its statistical uncertainty. The open circles correspond to MADGRAPH with the PYTHIA Z2* tune, the open squares to MADGRAPH with the PYTHIA Perugia 2011 tune, and the open triangles represent MADGRAPH with the PYTHIA Perugia 2011 noCR tune. The open diamonds correspond to POWHEG with the PYTHIA Z2* tune and the open crosses correspond to POWHEG with HERWIG 6 . The filled stars are for MC@NLO with HERWIG 6 and the open stars are for SHERPA.

\section{Alternative measurements of $\mathbf{m}_{t}$}

While the standard methods, mentioned before, provide the most precise measurement of the top quark mass, the results still suffer from systematic uncertainties mainly depending on the response of the jets, as well as on the modeling of the $\bar{t} \bar{t}$ system. Thus, aiming to improve these measurements, alternative methods, that use the relation of the top quark with an alternative observables sensitive to the top quark mass, are used. The latest CMS analyses that use alternative methods to measure the $\mathrm{m}_{\mathrm{t}}$ are summarized in Fig. 4 [15, 16, 17, 18, 19, 20, 21] and the results of some of them are discussed below.

\section{1 $\mathrm{m}_{\mathrm{t}}$ using single top quark events in proton-proton collisions events at $8 \mathrm{TeV}$}

Most analyses, aiming to measure the top quark mass, utilize samples of $\bar{t} \bar{t}$ events, since due to QCD processes top quarks are mainly produced in pairs. However, despite the lower rate of single top quark production occuring via weak interactions, determining of the top quark mass in these events com-

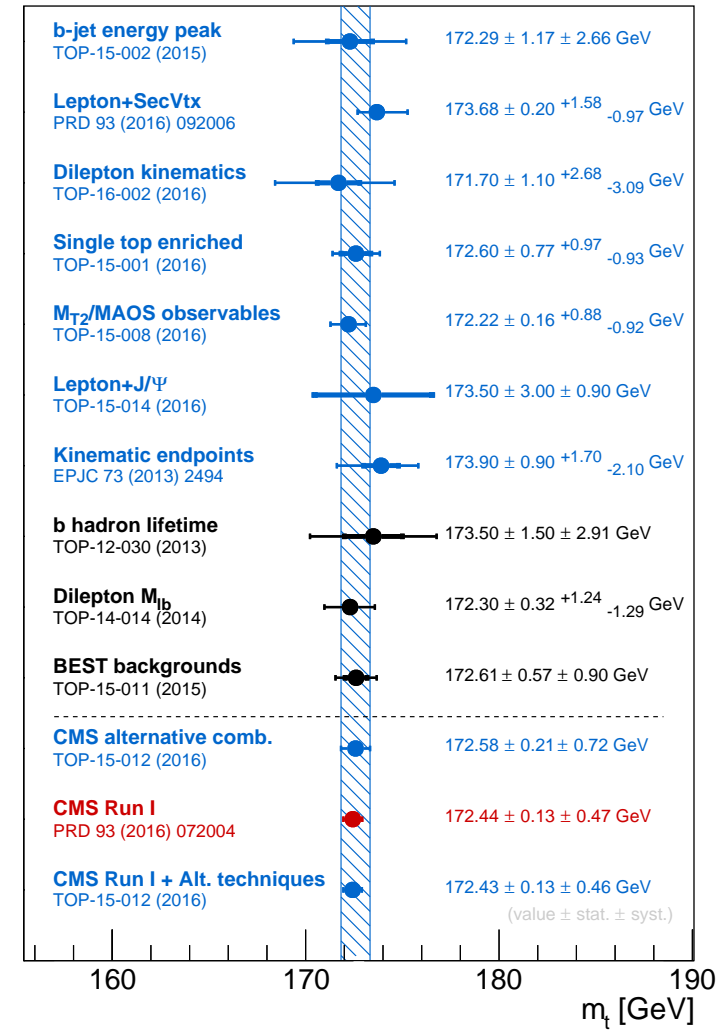

Figure 4: Summary of the new CMS mass measurements and the results derived from their combination [15]. 

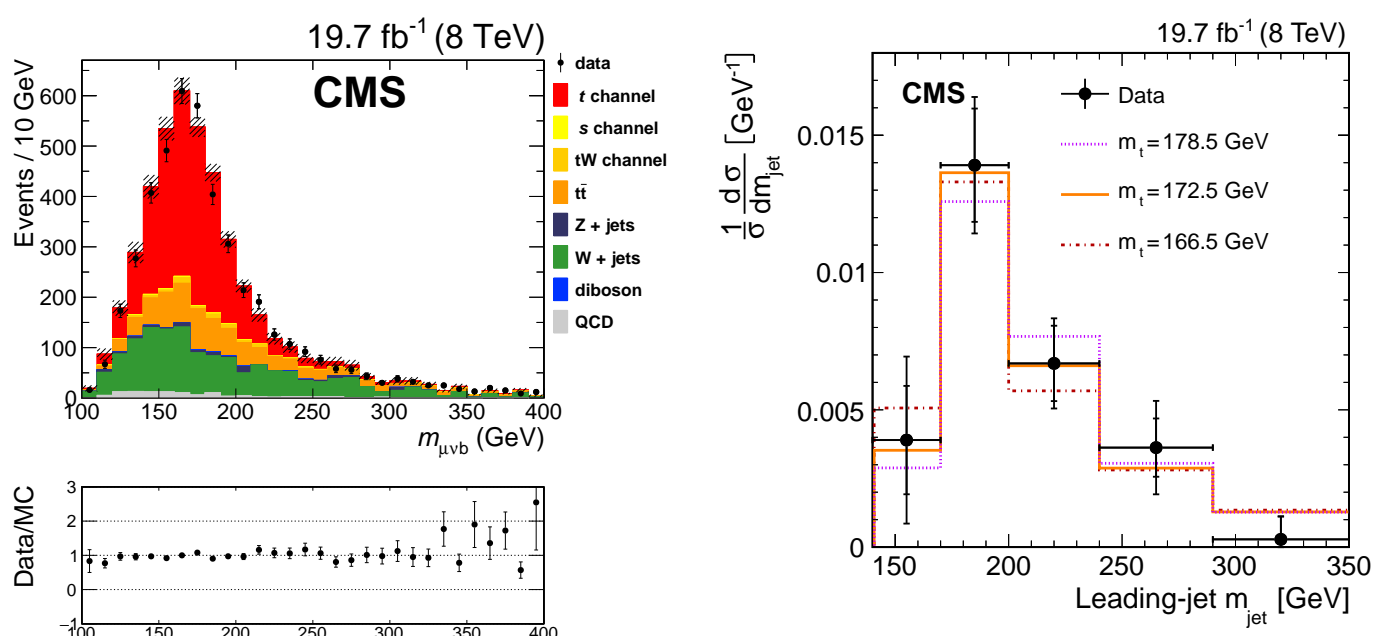

Figure 5: Reconstructed $\mu v \mathrm{~b}$ invariant mass distribution for data (points with error bars) and Monte Carlo events (stacked histograms) [16]. Final selection after the charge and light-quark jet pseudorapidity requirements. The ratio of the observed number of events in data to the number predicted by simulation is shown in the lower plots. The hatched area represents the uncertainty on the Monte Carlo predictions associated to the finite size of the samples and their normalization, and the integrated luminosity (left). The normalised particle-level $t \bar{t}$ differential cross section in the fiducial region as a function of the leading-jet mass [17]. The measurement is compared to predictions from MADGRAPH+PYTHIA for three values of $m_{\mathrm{t}}$. The vertical bars represent the statistical (inner) and the total (outer) uncertainties. The horizontal bars show the bin widths (right).

plements already available measurements. These measurements show less ambiguities in the jetparton assignment and have a different production mechanism, which can help to check for systematic biases in t⿱t due to the modelling of non-perturbative QCD effects. One of the recent CMS result [16] is a good example, where a tight selection is used to enrich the signal. The presence of the one isolated $\mu$ and one b-tagged jet are required. The selected single top t-channel events usually are accompanied by an additional light quark jet from the hard-scattering, it is measured in the forward region of $|\eta|>2.5$. Aiming to obtain as pure a sample as possible, only positively charged muons are selected, since in single-top production more top quarks than anti-quarks are produced at the LHC. Additionally, to suppress the background from QCD multijet, the tranverse mass of the $\mathrm{W}$ boson is required to be $m_{\mathrm{T}}(\mathrm{W})>50 \mathrm{GeV}$. As a result, the events are selected with 73 $\%$ purity and the distribution of the reconstructed $\mu \nu \mathrm{b}$ invariant mass is shown in the Fig. 5. The top quark mass is extracted from this distribution. The mass calibration from the fits to the set of simulated samples with different generated top quark mass is used in order to extract an unbiased value of $\mathrm{m}_{\mathrm{t}}$. The final result is $\mathrm{m}_{\mathrm{t}}=172.95 \pm 0.77$ (stat) $\pm_{0.93}^{0.97}$ (syst) GeV. It is the most precise $\mathrm{m}_{\mathrm{t}}$ extraction from single-top enriched data. The dominant systematic uncertainties for this analysis stem from the jet energy scale, the fit calibration, and the background modeling.

\section{$3.2 \mathrm{~m}_{\mathrm{t}}$ from boosted top jets $8 \mathrm{TeV}$}

The first measurement of the invariant mass of jets containing all decay products of the hadronically decaying top quark, namely a boosted top jet, presented recently by the CMS collaboration [17]. The analysis is performed using a sample of $t \bar{t}$ events with one muon or electron and two very energetic jets, one originates from the fragmented b quark and the other is a boosted top 
jet with the decay $\mathrm{t} \rightarrow \mathrm{Wb} \rightarrow \mathrm{qq}$ 'b. At high transverse momentum of the top quark, the products of the fully hadronicly decaying top quark are collimated and grouped into the single jet using the Cambridge-Aachen algorithm with a distance parameter $\mathrm{R}=1.2$. A transverse momentum of $400 \mathrm{GeV}$ is required for the leading jet. The jet originating from the $\mathrm{b}$ quark is required to have $\mathrm{p}_{\mathrm{T}}>150 \mathrm{GeV}$. In addition, a veto for additional jets with $\mathrm{p}_{\mathrm{T}, \text { veto }}>150 \mathrm{GeV}$ is applied. The mass of the top jet is calculated using four-vectors of all particles clustered into the jet. A fiducial region with merged top quark decays in hadronic final states is used to perform the measurement. The corrections for detector effects are applied using a regularized unfolding. To extract a value of the $\mathrm{m}_{\mathrm{t}}$ in the boosted regime, the normalized particle-level $\mathrm{t} \overline{\mathrm{t}}$ cross section of the leading jet mass is used, as shown on the Fig. 5. The obtained value is found to be $\mathrm{m}_{\mathrm{t}}=170.8 \pm 6.0$ (stat) \pm 2.8 (syst) \pm 4.6 (mod) \pm 4.0 (theo) $\mathrm{GeV}$, which demonstrate the feasibility of the method to measure the $\mathrm{m}_{\mathrm{t}}$ in the highly boosted regime.

\section{Summary}

In this work some of the latest results on the top quark mass are discussed. The measurements using standard methods still provide the most precise value with $\mathrm{m}_{\mathrm{t}}=172.44 \pm 0.13$ (stat) \pm 0.47 (syst) $\mathrm{GeV}$. This result is confirmed at $13 \mathrm{TeV}$. Meanwhile, the results coming from alternative measurements, such as the $\mathrm{m}_{\mathrm{t}}$ measured using single-top events, achieve a comparable precision. The combination of the CMS alternative technique measurements of the top quark mass has a value of $\mathrm{m}_{\mathrm{t}}=172.58 \pm 0.21$ (stat) \pm 0.72 (syst) GeV. In addition, we have presented the first measurement of $m_{t}$ using boosted top jets, which gives the proof-of-principle for a new determination method.

\section{References}

[1] CMS Collaboration. The CMS Experiment at the CERN LHC. JINST, 3:S08004, 2008.

[2] CMS Collaboration. Measurement of the top quark mass using proton-proton data at $\sqrt{s}=7$ and 8 TeV. Phys. Rev. D, 93:072004, 2016.

[3] ATLAS, CDF, CMS, D0 Collaborations. First combination of Tevatron and LHC measurements of the top-quark mass. ATLAS-CONF-2014-008, CDF-NOTE-11071, CMS-PAS-TOP-13-014, D0-NOTE-6416, 2014, arXiv:1403.4427.

[4] S. Moch et al. High precision fundamental constants at the TeV scale. DESY-14-081, MITP-14-036, 2014, arXiv:1405.4781.

[5] Peter Marquard, Alexander V. Smirnov, Vladimir A. Smirnov, and Matthias Steinhauser. Quark Mass Relations to Four-Loop Order in Perturbative QCD. Phys. Rev. Lett., 114:142002, 2015.

[6] Gennaro Corcella. Interpretation of the top-quark mass measurements: a theory overview. In Proceedings, 8th International Workshop on Top Quark Physics (TOP2015): Ischia, Italy, September 14-18, 2015, volume TOP2015, page 037, 2016.

[7] M. Beneke, P. Marquard, P. Nason, and M. Steinhauser. On the ultimate uncertainty of the top quark pole mass. DESY-16-082, TTP16-016, TUM-HEP-1044-16, 2016, arXiv:1605.03609.

[8] Mathias Butenschoen, Bahman Dehnadi, Andre H. Hoang, Vicent Mateu, Moritz Preisser, and Iain W. Stewart. Top Quark Mass Calibration for Monte Carlo Event Generators. Phys. Rev. Lett., 117(23):232001, 2016. 
[9] CMS Collaboration. Measurement of the top quark mass with muon+jets final states in pp collisions at $\sqrt{s}=13 \mathrm{TeV}$. CMS Physics Analysis Summary CMS-PAS-TOP-16-022, 2016.

[10] Victor Mukhamedovich Abazov et al. Precision measurement of the top-quark mass in lepton+jets final states. Phys. Rev. D, 91:112003, 2015.

[11] CMS Collaboration. Measurement of the top-quark mass in $t \bar{t}$ events with lepton+jets final states in $p p$ collisions at $\sqrt{s}=7 \mathrm{TeV}$. JHEP, 12:105, 2012.

[12] T. Aaltonen et al. Precision Top-Quark Mass Measurements at CDF. Phys. Rev. Lett., 109:152003, 2012.

[13] Georges Aad et al. Measurement of the top quark mass in the $t \bar{t} \rightarrow$ lepton+jets and $t \bar{t} \rightarrow$ dilepton channels using $\sqrt{s}=7 \mathrm{TeV}$ ATLAS data. Eur. Phys. J. C, 75:330, 2015.

[14] R. H. Dalitz and Gary R. Goldstein. The Decay and polarization properties of the top quark. Phys. Rev., D45:1531-1543, 1992.

[15] CMS Collaboration. Combinations of the CMS alternative technique measurements of the top quark mass. 2016.

[16] CMS Collaboration. Measurement of the top quark mass using single top quark events in proton-proton collisions at $\sqrt{s}=8 \mathrm{TeV}$. Eur. Phys. J. C, 77(5):354, 2017.

[17] CMS Collaboration. Measurement of the jet mass in highly boosted t $\bar{t}$ events from pp collisions at $\sqrt{s}=8$ TeV. Eur. Phys. J. C, 77(7):467, 2017.

[18] CMS Collaboration. Measurement of the mass difference between top quark and antiquark in pp collisions at $\sqrt{s}=8 \mathrm{TeV}$. Phys. Lett. B, 770:50-71, 2017.

[19] CMS Collaboration. Measurement of the $t \bar{t}$ production cross section using events with one lepton and at least one jet in pp collisions at $\sqrt{s}=13 \mathrm{TeV}$. JHEP, 09:051, 2017.

[20] CMS Collaboration. Measurement of the top quark mass in the dileptonic $t \bar{t}$ decay channel using the mass observables $M_{b \ell}, M_{T 2}$, and $M_{b \ell v}$ in pp collisions at $\sqrt{s}=8 \mathrm{TeV}$. Phys. Rev. D, 96(3):032002, 2017.

[21] CMS Collaboration. Measurement of the mass of the top quark in decays with a $J / \psi$ meson in pp collisions at $8 \mathrm{TeV}$. JHEP, 12:123, 2016. 